\title{
Periodicity of GABA-Containing Cells in Primate Prefrontal Cortex
}

\author{
Michael L. Schwartz, De-Shu Zheng, and Patricia S. Goldman-Rakic \\ Section of Neuroanatomy, Yale University School of Medicine, New Haven, Connecticut 06510
}

\begin{abstract}
The tangential distribution of GABA-containing cells was examined in the principal sulcus of the frontal lobe in 12 macaque monkeys. Following immunostaining with GABA antisera all immunoreactive cells were charted and their distribution analyzed with both statistical and spectral density methods. In addition, a gapless series of sections was used to generate a 2-dimensional reconstruction of cell disposition in the tangential plane parallel to the pia. Our findings indicate that the GABA cells are not distributed uniformly across the cortex, as is commonly believed, but that their density is characterized by 2 independent sinusoidal fluctuations: a high-frequency component with a period ranging from 150 to $250 \mu \mathrm{m}$ superimposed upon a lower-frequency component with a period of 1000-1275 $\mu \mathrm{m}$. The half-cycle of the low-frequency component (roughly $625 \mu \mathrm{m}$ ) is very similar to the dimensions of afferent and efferent columns in the principal sulcus, while the half-cycle of the higherfrequency component (approximately $125 \mu \mathrm{m}$ ) is closer in size to that of the functionally defined columns of neurons found in regions of sensory cortex that share common physiological properties. To our knowledge, these findings are the first indication that inhibitory local circuit neurons are not uniformly or randomly distributed, but exhibit periodicities that may be related to the columnar, functional and architectural organization of the cortex.
\end{abstract}

Studies using retrograde and anterograde tract-tracing methods have revealed a periodic organization of cortical afferents and efferents (Jones et al., 1975, 1978; Goldman and Nauta, 1977; Hedreen and Yin, 1981; Killackey et al., 1983; Schwartz and Goldman-Rakic, 1984; Andersen et al., 1985; Caminiti et al., 1985). The units of this spatially periodic pattern have been variously described as columns, clusters, or modules, and appear as areas of dense cell or terminal label separated by regions free of, or with reduced densities of, label in tract-tracing studies. Moreover, studies of diverse cortical afferents of the prefrontal association (Goldman-Rakic and Schwartz, 1982) and somatosensory (Jones et al., 1979) cortices have demonstrated that the terminal columns originating from contralateral and ipsilateral cortical regions interdigitate. A similar organizational relationship holds for the efferent cell populations projecting to the same and opposite hemispheres (Schwartz and Goldman-

\footnotetext{
Received Feb. 7, 1987; revised July 14, 1987; accepted Sept. 27, 1987

The authors wish to thank Marianna Pappy and JoAnn Coburn for technical assistance. This work was supported by NIH and NIMH Grants POI-NS 22807 and ROl MH38546.

Correspondence should be addressed to Michael L. Schwartz, Ph.D., Section of Neuroanatomy, Yale University School of Medicine, 333 Cedar Street, SHM, New Haven, CT 06510.

Copyright (C) 1988 Society for Neuroscience $0270-6474 / 88 / 061962-09 \$ 02.00 / 0$
}

Rakic, 1984). Modular organization of afferent and efferent territories in cortex is also evident in descending projection systems. Corticothalamic, corticopontine, corticobulbar, and corticospinal neurons all reveal a similarly disjunctive or clustered arrangement (Jones and Wisc, 1977; Arikuni ct al., 1983; Giguere and Goldman-Rakic, 1985). These repetitive anatomical arrangements of different cortical areas and major efferent and afferent populations of the cortex suggest that the column is a basic unit of cortical organization and information processing (Mountcastle, 1978; Eccles, 1984; Goldman-Rakic, 1984).

In contrast to the detailed knowledge about the modular organization of extrinsic cortical connections, relatively little is known about the tangential distribution of local circuit neurons of the cortex. In part, this may be due to methodological constraints. For example, until recently, most of our knowledge of intrinsic cortical neurons was based on studies using the Golgi impregnation technique. Although providing significant information on the morphology and laminar distribution of intrinsic neurons, Golgi studies have been less useful for the analysis of tangential variations. Modern immunocytochemical methods for analyzing neuronal populations offer new opportunities for examining this issue (for review, see Jones, 1984). GABA is the principal neurotransmitter used by the vast majority of local circuit neurons (Krnjevic, 1974; Emson and Lindvall, 1979), and studies using antisera against both GABA and its synthetic enzyme glutamic acid decarboxylase (GAD) have clearly demonstrated laminar variations in the density of this inhibitory interneuron in a number of cortical areas and species (Ribak, 1978; Hendrickson et al., 1981; Houser et al., 1983; Schwartz et al., 1985; Gabbott and Somogyi, 1986). In the present study immunocytochemical methods have been used to demonstrate that the density of GABA-containing cells also varies tangentially across the cortical surface.

\section{Materials and Methods}

Subjects and procedures. Tissue from the principal sulcal cortex (area 46 of Walker, 1940; see Fig. 1) of 12 macaque monkeys, 3 cynomolgus $(M$. fascicularis) and 9 rhesus ( $M$. mulatta), was processed for the immunocytochemical visualization of GABA. One of the cynomolgus monkeys received three $0.3 \mu \mathrm{l}$ injections of colchicine $(6 \mu \mathrm{g} / \mu \mathrm{l}$ in physiological saline) into the dorsal bank of the principal sulcus $24 \mathrm{hr}$ prior to being killed, to enhance the staining of cell bodies (Freund et al., 1983). However, the pattern of immunocytochemical staining for this monkey was not appreciably different than that of the other animals in this series. All animals were deeply anesthetized using sodium pentobarbital and were then perfused through the heart with an initial flush of PBS, pH 7.4, followed by 1-2 liters of fixative. Several fixatives were used to compare the quality of staining and to insure that the results obtained with a given fixative did not introduce any bias into the pattern of immunocytochemical staining. Among the fixatives used were: (1) $5 \%$ acrolein in PBS (3 monkeys); (2) 4\% paraformaldehyde, using the low-high pH protocol of Berod et al. (1981) (3 monkeys); (3) 4\% paraformaldehyde $+0.1 \%$ glutaraldehyde in PBS ( 2 monkeys); and (4) $4 \%$ 


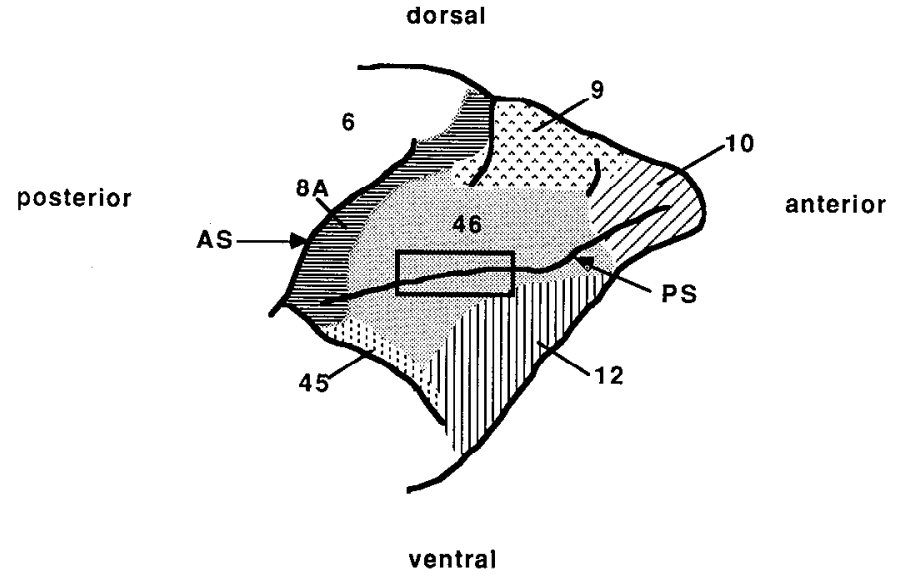

Figure 1. A. E. Walker's (1940) cytoarchitectonic map of the lateral surface of the macaque prefrontal cortex. The rectangular area overlying the middle portion of the principal sulcus $(P S)$ represents the region of area 46 , which was dissected out and immunohistochemically processed for the visualization of GABA-like immunoreactivity. Numbers correspond to Walker's designations for different cytoarchitectonic areas of macaque prefrontal cortcx. $A S$, arcuatc sulcus.

paraformaldehyde in PBS (4 monkeys). While the laminar pattern and morphology of GABA-immunoreactive cells was similar for each of the fixatives used, there were clear differences in the quality and overall density of cells stained. Brains from monkeys fixed with $5 \%$ acrolein yielded the most intense pattern of neuronal staining, although the background staining in these brains was always greater than was found with the other fixatives (Fig. 2, $A, C$ ). Fixation with $4 \%$ paraformaldehyde and the low-high pH protocol (Fig. $2 B$ ) or with $4 \%$ paraformaldehyde + $0.1 \%$ glutaraldehyde yielded comparable staining with clear backgrounds. Brains from monkeys perfused with $4 \%$ paraformaldehyde in PBS were clearly inferior in the staining of GABA-immunoreactive cells and were only examined qualitatively. Following perfusion, the brains were removed from the cranium and blocked. Blocks of tissue were taken from the dorsal and ventral banks of the principal sulcus. These were stored in PBS for 2-16 hr and then Vibratome-sectioned in the coronal plane at $50 \mu \mathrm{m}$. All sections were cut into cold $0.05 \mathrm{~m}$ phosphate buffer ( $\mathrm{PB} ; \mathrm{pH} 7.2)$. To insure that variations in the density of immunostained cells were not merely a reflection of cutting "chatter" in Vibratome-sectioned tissue, the orientation of the blade to the tissue block was varied from monkey to monkey and from block to block in monkeys from which more than one block was taken. For example, in some blocks the blade sectioned across the cortex of the principal sulcus from lateral to medial, while other blocks were sectioned going from dorsal to ventral, i.e., parallel to the laminae of the principal sulcal cortex. As a further control for potential biases introduced by vibratome sectioning of the tissue, additional blocks from 2 monkeys were frozen-sectioned at the same thickness. Finally, in one rhesus monkey, every section in a gapless series of 26 sections was saved and processed in order to constuct a 2-dimensional representation of the pattern of GABA-like neuronal staining.

Immunohistochemistry. Incubations for the immunocytochemical visualization of GABA were carried out on free-floating sections as follows: 7 washes in $0.1 \mathrm{M} \mathrm{PB} \mathrm{(2-5} \mathrm{hr),} 30 \mathrm{~min}$ in $5 \%$ normal goat serum (NGS) in $\mathrm{PB}, 16-24 \mathrm{hr}$ at $4^{\circ} \mathrm{C}$ in rabbit anti-GABA (Immuno-Nuclear) diluted $1: 2500$ or $1: 5000$ in 5\% NGS and PB, 7 washes in PB, 90 min in biotinylated goat anti-rabbit $\operatorname{IgG}$ (Vector), 7 washes in PB, $45 \mathrm{~min}$ in HRP avidin D (Vector), and 5 washes in $0.05 \mathrm{M}$ Tris buffer. The sections were then preincubated for $5-10 \mathrm{~min}$ in $0.05 \%$ of $3,3^{\prime}$-diaminobenzidine tetra $\mathrm{HCl}$ in Tris buffer, followed by a $5-15 \mathrm{~min}$ incubation in a fresh volume of the same solution containing $0.03 \%$ hydrogen peroxide. They were then washed 4 times in Tris buffer, mounted onto subbed slides, dehydrated, and coverslipped. To enhance the penetration of the primary and secondary antisera, some sections from each brain had $0.2-0.3 \%$ Triton $\mathrm{X}-100$ added to the incubation solutions.

The antiserum to GABA was generated in rabbit using GABA conjugated to bovine serum albumin and was obtained from ImmunoNuclear. A detailed characterization of the antiserum has been reported by Maley and Newton (1985). To check the specificity of staining in our material, several controls were used. Normal rabbit serum was substituted for anti-GABA in the primary antisera incubation, or tissue was incubated in anti-GABA that had been preabsorbed with GABA (Sigma; $100 \mu \mathrm{g} / \mathrm{ml}$ ). In neither case was specific staining of cell bodies or terminals observed.

Chi-square and spectral analysis. All immunocytochemically reacted sections were examined qualitatively and then 2-10 sections from each of 8 monkeys perfused with fixatives 1-3 (see Subjects and Procedures, above) were analyzed quantitatively. For each animal, a $2.0-4.0-\mathrm{mm}-$ wide region was selected from either the dorsal or ventral bank of the PS, and every cell with GABA-like immunoreactivity was charted using a Lietz Dialux microscope (with $12.5 \times$ eyepieces and a $16 \times$ waterimmersion objective) coupled to an $X-Y$ plotter. The length of the segment charted in each section was restricted to regions of roughly uniform thickness (from the pia to the white matter border) and levelness, thus guaranteeing minimal displacement of cells in the horizontal plane of curvature. These criteria limited the number and size of regions that could be analyzed in the invaginated cortex of the principal sulcus (PS), but were considered essential to insure an unbiased evaluation of the cell distribution. Charts of immunoreacted cells were then entered into a computer using a digitizing tablet and subjected to a "bin analysis" that divided the cortex into bins of arbitrary width, spanning all cortical layers and oriented perpendicular to the pial surface (see Fig. 3). The number of immunoreactive neurons in each bin was then determined and a histogram of the distribution was generated. To insure that the distribution of cells with GABA-like immunoreactivity was not biased by the choice of bin width, the data for each section were analyzed using several different bin widths, ranging from 50 to $500 \mu \mathrm{m}$.

Histograms generated by the bin analysis were first examined by eye to determine whether the distribution of GABA-containing cells exhibited periodicity. Several sections with optimal immunostaining were then selected from 5 monkeys for further statistical evaluation. These were first evaluated using a chi-square $\left(\chi^{2}\right)$ test to assess the uniformity of the GABA cell distribution, and then were subjected to a spectral density analysis to investigate the periodicity of the distribution. The spectral density or frequency domain analysis is a descriptive statistic that assumes that a data series may be represented by the summation of a series of sine waves of different frequencies. This statistic provides a means of assessing the relative contribution of each of the different component frequency bands to the variation of the overall distribution. The method performs a Fourier transformation of the data to provide coefficients for the sinusoidal components representing the distribution at a discrete set of frequencies. An estimated spectral density curve is then generated by computing the average squared amplitude of the sinusoids for each frequency band; these values are then plotted as a function of frequency, thus forming a periodogram (Thrall and Engelman, 1983). Most analyses were performed on histograms with a bin width of $50 \mu \mathrm{m}$, although some histograms with a bin width of 25 or $100 \mu \mathrm{m}$ were also examined.

Serial reconstruction. In one monkey, the ventral bank of the middle third of the PS was removed, sectioned at $50 \mu \mathrm{m}$, and a gapless series of 26 sections was then processed for GABA immunocytochemistry. From this larger series, a smaller group of 8 consccutive sections met the criteria for density and uniformity of GABA-like reactivity. In this series, all cells with GABA-like immunoreactivity and containing a nucleolus, were plotted for a 2-3-mm-long segment of the ventral bank of the PS. Landmarks such as blood vessels were also plotted, so that successive sections could be aligned using a minimum of 3 shared landmarks. These sections were then subjected to a bin analysis (bin width $=$ $50 \mu \mathrm{m})$ as previously described, and the results were used to create a 2-dimensional reconstruction of the density of GABA-like cells across the cortex (see Fig. 8). Finally, bins containing more (or fewer) than the mean number of GABA-immunoreactive cells were connected (shaded area in Fig. 8).

\section{Results}

\section{Quantitative bin analysis of individual sections}

Visual examination of plots of GABA-immunoreactive cells indicates that this neuronal population is distributed throughout all cortical layers, though the density of cells is greatest in 2 bands: one in layer IV and a second that includes superficial layer III and layer II (Schwartz et al., 1984). Although, within 

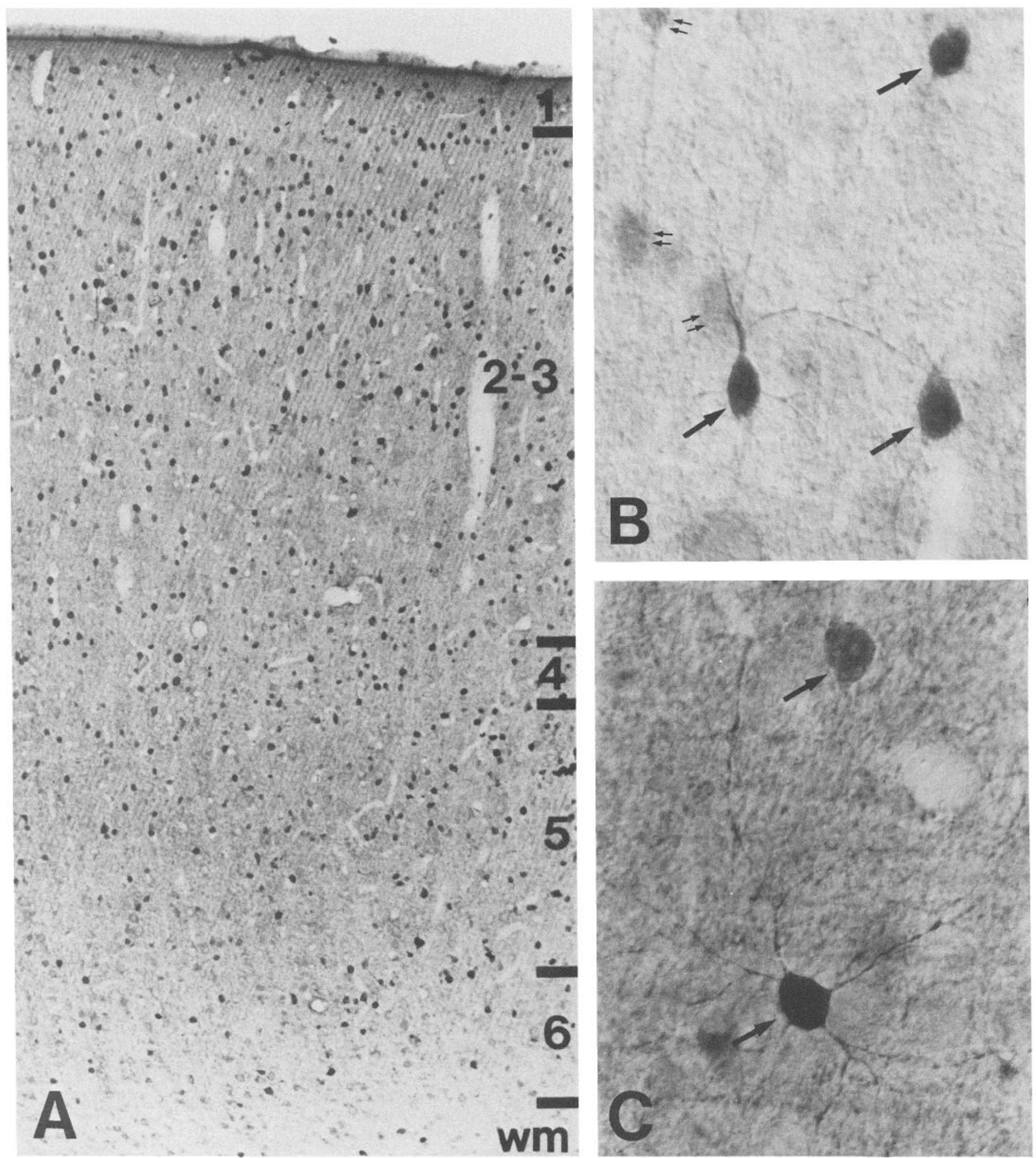

Figure 2. GABA-immunoreactive neurons in area 46 of the monkey prefrontal cortex. $A$, Low-power micrograph showing the pattern of staining for all cortical layers. The intense staining of cell bodies is characteristic of the monkeys perfused with $5 \%$ acrolein. $B$, High-magnification micrograph illustrating the labeling of GABA-immunoreactive neurons in layer III of a monkey perfused with $4 \%$ paraformaldehyde and the low-high pH protocol of Berod et al. (1981). Large arrows mark cells labeled in the plane of focus and small double arrows indicate labeled cells at a different depth of focus. $C$, GABA-immunoreactive cells in layer IV and lower layer III from a monkey perfused with acrolein. 


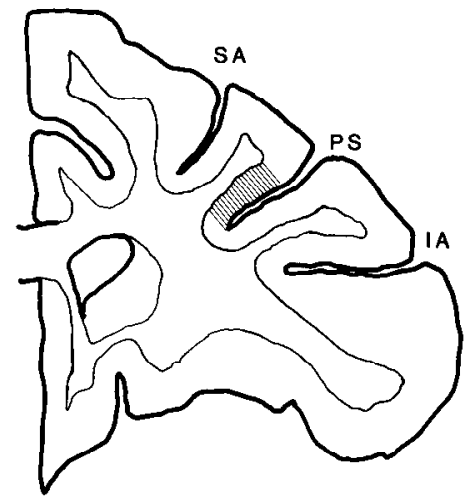

Figure 3. Coronal section of the monkey prefrontal cortex. A schematic representation of the division of cortex into bins is shown for the dorsal bank of the principal sulcus (PS). Equal-sized bins are oriented perpendicular to the pial surface and span all 6 cortical layers. $S A$, superior limb of the arcuate sulcus; $I A$, inferior limb of the arcuate sulcus.

layers, cells were frequently observed in small clusters or in strings (Figs. 2, 4), they nevertheless appeared to have a uniform distribution in the tangential plane. In spite of this visual impression, however, histograms of $50 \mu \mathrm{m}$ bin width showed that GABA-like cells cluster into areas of high density spanning 100$150 \mu \mathrm{m}$ (2-3 bins; Fig. 5). Varying the bin width altered the number of bins in a cluster, but not the width of the area containing a high (or low) density of GABA-like cells. A lower frequency of variation was also observed in most histograms. This component of the distribution appeared as a sinusoidal variation in the basal level of GABA-like cells upon which the higher-frequency component was superimposed. The low-frequency component appeared as a clustering of GABA-like cells into alternating regions of high and low density measuring approximately $300-600 \mu \mathrm{m}$ in width; this pattern was most visible in histograms with bin widths exceeding $100 \mu \mathrm{m}$ (these effectively filtered out the high-frequency component of variation; Fig. 6).

\section{Chi-square $\left(x^{2}\right)$ and spectral density analyses}

To test the hypothesis that the distribution of cells with GABAlike immunoreactivity is uneven, we subjected the binned data to independent chi-square tests for uniformity. For this analysis the data for cach histogram were compared to a histogram of uniform distribution with a mean value equal to that of the data histogram. The results of these statistical tests yielded highly significant values, allowing rejection of the null hypothesis that the pattern of cell labeling was uniform. All 24 sections examined in this manner yielded highly significant $\chi^{2}$ values, with probabilities of $p \leq 0.001$. The range of values was $\chi^{2}=106.7-$ 505.7. For example, the data in Figure 5 yielded $\chi^{2}=505.7$, $d f=75, p \leq 0.001$.

To evaluate whether the lack of uniformity described by the $\chi^{2}$ test was due to a random or periodic distribution, a spectral analysis using Fourier transformations was then carried out on selected sections (Thrall and Engelman, 1983). The bin data from each section were first analyzed without any smoothing or filtering and were then reanalyzed following smoothing with an autoregressive filter estimated from the data set. For each periodogram, the width of a peak indicates how sharp the periodicity is, a wide peak representing a greater range in the value of the period, while a narrow peak represents a more sharply periodic distribution. The height of a peak at a given frequency, on the other hand, represents the "power" or the relative contribution of a sinusoidal component at that frequency to the overall pattern of cell distribution. In the present study we report ranges of peak values for periodicities that were common to all sections and animals analyzed. Although the periodograms of individual sections typically contained peaks at several frequencies, all sections analyzed by this technique consistently contained peaks in 2 particular frequency ranges: a high-frequency component with a peak power in the range of $150-250 \mu \mathrm{m} / \mathrm{cycle}$, and a low frequency component with a peak power between 1000 and $1275 \mu \mathrm{m} /$ cycle (Fig. 7). In addition to these 2 principal peaks, a third peak in the very-high-frequency range was evident in a majority, but not all, of the sections with bin widths of $50 \mu \mathrm{m}$. This component represented a periodicity of approximately 90 $125 \mu \mathrm{m} /$ cycle (Fig. 7, B, C). Whether this value represents a

$1 \mathrm{~mm}$

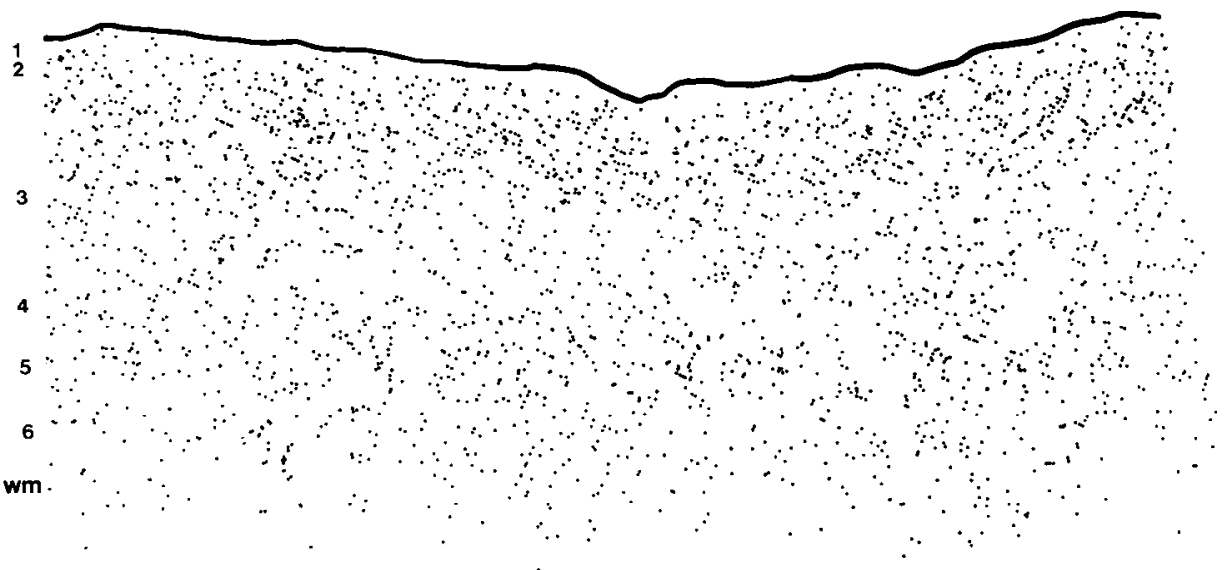

Figure 4. Charting of all neurons containing GABA-like immunoreactivity in a $50-\mu \mathrm{m}$-thick section from a portion of Walker's area 46 within the ventral bank of the principal sulcus. $P S$, principal sulcus; $w m$, white matter. 
Figure 5. Tangential distribution of immunoreactive neurons in a $50-\mu \mathrm{m}$ thick section from the ventral bank of the PS. The number of cells with GABAlike reactivity in adjacent $50 \mu \mathrm{m}$ bins oriented perpendicular to the pial surface, and spanning all layers, are represented in this histogram. The distance in micrometers is represented starting from an arbitrary point near the fundus of the sulcus and proceeding laterally. The distribution appears to have a highis superimposed upon a lower-frequency component. Asterisks represent bins in which large, penetrating vessels were found at the surface of the section. frequency component of variation that

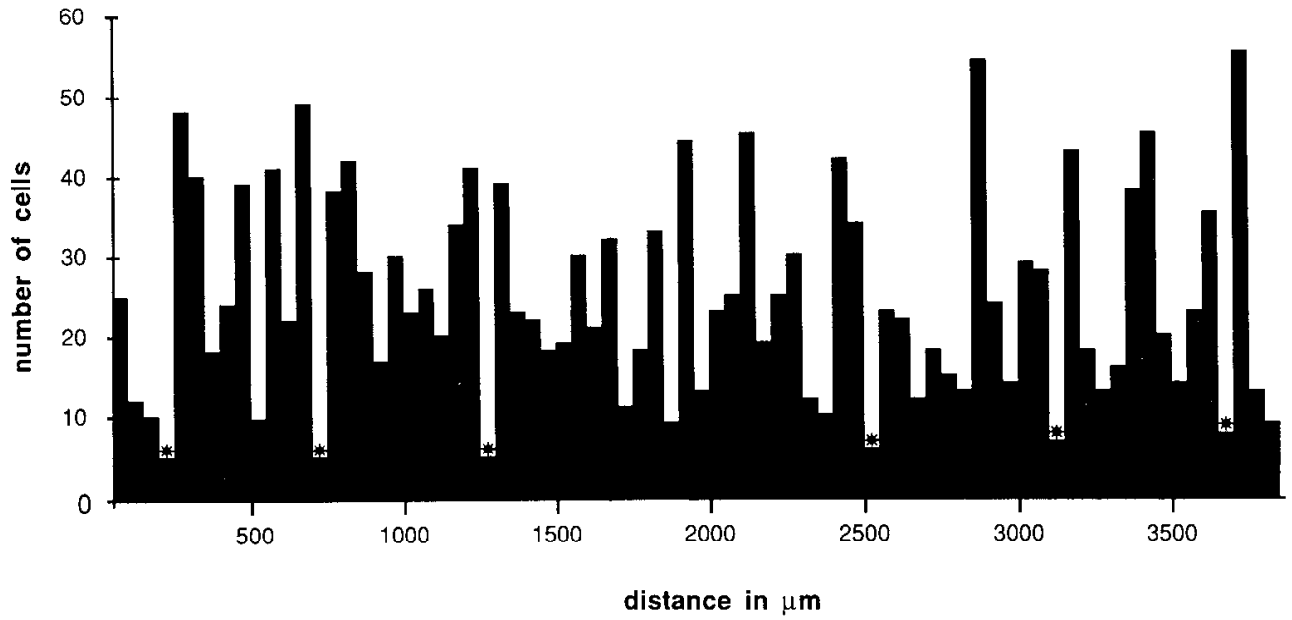

third component of variability or is an artifact cannot be determined from our analysis and remains to be more fully examined in future studies.

\section{Serial reconstruction}

An additional analysis to assess the 2-dimensional distribution of cells with GABA-like immunoreactivity was carried out using serial section reconstruction methods for a region of area 46 (Fig. 8). The reconstruction is of eight $50-\mu \mathrm{m}$-thick serial sections from the ventral bank of the PS. The number of cells in each 50- $\mu$ m-wide bin is shown. Bins containing GABA cell numbers in excess of the mean number of GABA cells per bin for the entire reconstruction were connected and are shown as the shaded area in Figure 8. This method showed clear variations in the density of cells with GABA-like immunoreactivity across the cortical surface. This reconstruction further reveals the mosaic organization of this distribution, with areas containing a high density of GABA-like cells interweaving with regions containing fewer cells. The more prominent pattern of variability in the reconstruction corresponds particularly well to the highfrequency component $(150-250 \mu \mathrm{m} /$ cycle) revealed in the spectral density analysis. To determine whether the disjunctive pattern could be attributed to the arbitrary criterion for high- and low-density bins, i.e., bins containing more or fewer than the mean number of cells, respectively, a second reconstruction was made from the same data, but shading was based on the presence Although this new criterion modified the number of alterations of high- and low-density regions, several features of the distribution remained invariant. Most notably, the remaining regions of high GABA cell density continued to be 1-2 bins in width, and these regions were frequently continuous across several sections, indicating that they were not merely artifacts of uneven immunohistochemical staining for an individual section, but represented a true organizational feature of this neuronal population.

The reconstruction of serial sections failed to clearly reveal a low-frequency $(1000-1250 \mu \mathrm{m} / \mathrm{cycle})$ component in the distribution despite the presence of this component in the spectral density analysis of the same data. The reasons for this are unclear but may include, among other factors, the difficulty in defining boundaries of a slowly moving sinusoid. This may be less of a problem with more rapidly varying components, which, by definition, have sharper peaks and valleys in their cell density.

\section{Discussion}

The present results provide evidence that GABA-containing neurons have an uneven distribution across the cortical surface, with regions containing a high density of GABA cells interweaving with regions containing a lower density of cells. The of regions that contained values at least $1 \mathrm{SD}$ above the mean.
Figure 6. Histogram of the tangential distribution of immunoreactive neurons taken from the same section shown in Figure 5. In this histogram, however, the bin size has been increased to 100 $\mu \mathrm{m}$ to filter out the high-frequency component of the distribution and to more clearly demonstrate variations in the low-frequency component.

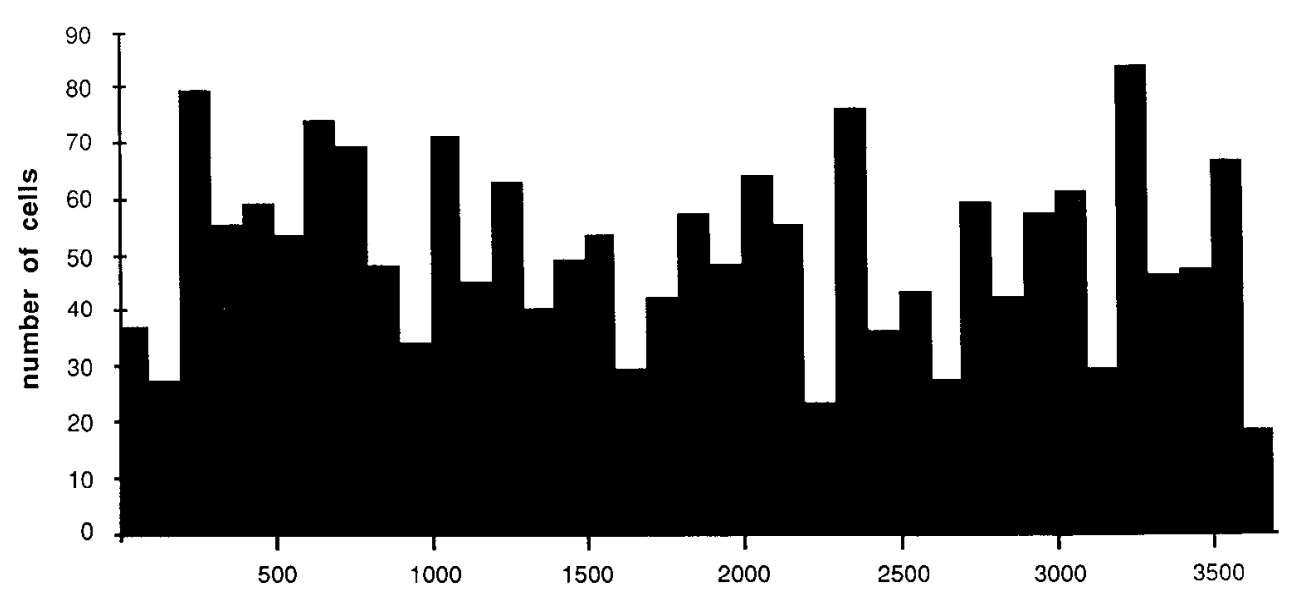

distance in $\mu \mathrm{m}$ 
A

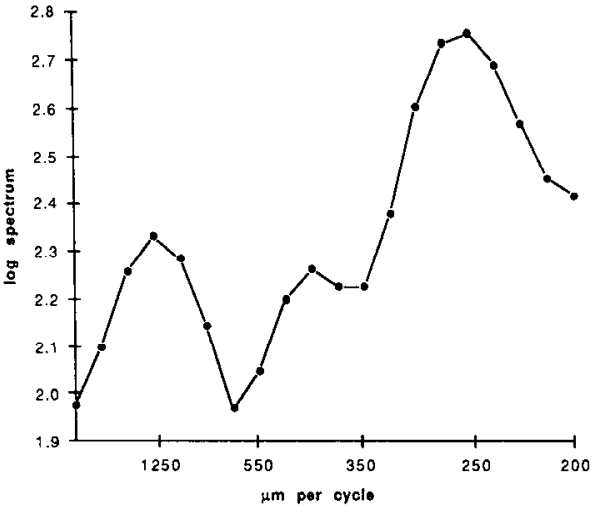

B

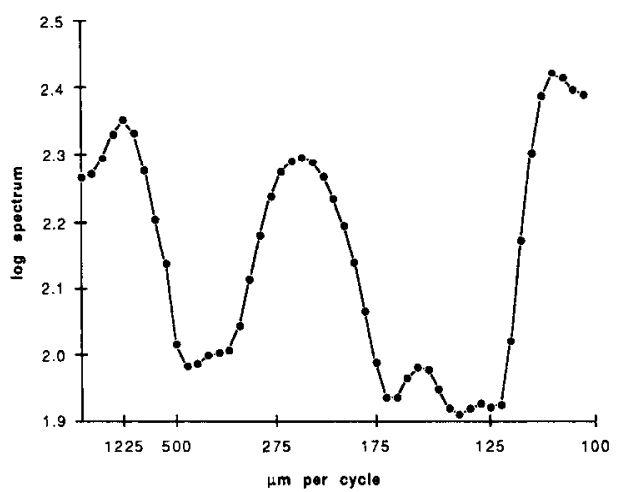

C

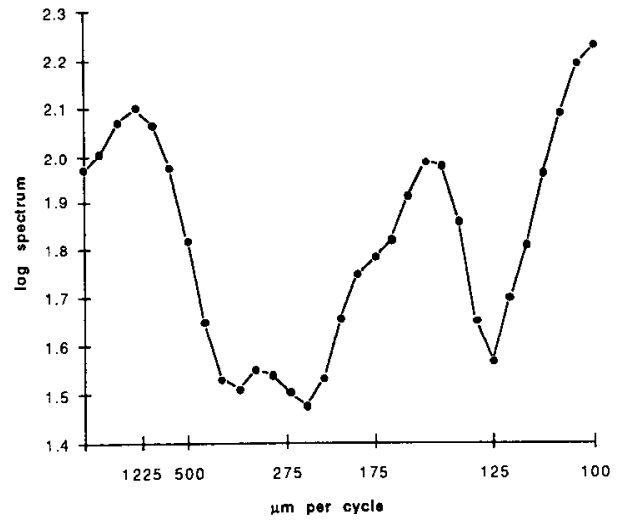

Figure 7. Spectral density curves of sections from 3 different monkeys. Each periodogram was smoothed using an autoregressive filter. $A$, Periodogram estimated from a histogram with a bin width of $100 \mu \mathrm{m}$. Two dominant peaks are present in the periodogram; one with a peak power at $1267 \mu \mathrm{m} /$ cycle and one at $253 \mu \mathrm{m} /$ cycle. $\Lambda$ cycle represents the width of an area containing adjacent regions of higher and lower GABAimmunoreactive cell density. Although other elevations are apparent in this distribution, only the peaks at the 2 frequencies noted were seen in every section analyzed in this manner. $B$, Periodogram estimated from a histogram with a bin width of $50 \mu \mathrm{m}$. Three peaks were found in this periodogram: one with a peak power at $1225 \mu \mathrm{m} / \mathrm{cycle}$, one at $222 \mu \mathrm{m} /$ cycle, and one at $106.5 \mu \mathrm{m} /$ cycle. The latter peak was seen in a majority of the histograms with a bin width of $50 \mu \mathrm{m}$; however, it remains to be determined whether this truly represents a third component of the distribution or is due to some artifact. $C$, Periodogram estimated from a histogram with a bin width of $50 \mu \mathrm{m}$. As in $B, 3$ prominent peaks are present: one at $1000 \mu \mathrm{m} /$ cyle, one at $160 \mu \mathrm{m}$, and a third elevation for which the peak power is not clear, but which lies at the extreme of the high-frequency portion of the periodogram. results of all 3 methods-bin analysis; statistical tests, including the $\chi^{2}$ test for uniformity and a spectral density analysis; and serial reconstruction-were congruent. Thus, one full cycle of the high-frequency component revealed by spectral density analysis contains 2 regions; an area of high GABA cell density and a region of lower density each corresponds to approximately one-half the territory of one full cycle, i.e., $126 \mu \mathrm{m}$. This value fits nicely with the high-frequency $(150-250 \mu \mathrm{m})$ clustering of bins of high and low density observed in histograms of the bin analysis. Similarly, when the value for the low-frequency component in Figure $7 A$ is broken down into its low- and highdensity constituents, a value of $634 \mu \mathrm{m}$ is obtained, which fits well with the independent measurements of 300 to $600 \mu \mathrm{m}$ obtained from the histograms. The relative contributions of the high- and low-frequency components (as determined by their peak power) to the overall distribution varied from animal to animal and, in some cases, between sections of the same monkey. However, in spite of this variability, these 2 peaks consistently appeared in all monkeys and in all sections examined with spectral density methods, thus indicating that these 2 peaks are a general organizational feature of GABA-containing cells. It should be emphasized that among the periodograms obtained from different animals there were other peaks that did not appear consistently. By focusing on those features of the organization of GABA-like cells that are shared across animals, we do not wish to rule out the possibility that other periodicities may also be significant for information processing within the cortex.

\section{Methodological issues}

As with all immunocytochemical studies, there are a number of potential sources of artifact that must be considered when drawing inferences about the organization of an immunoreactive population. In the present study we have identified at least 2 factors that could bias the distribution of GABA-like immunoreactivity. These may be generally categorized as sectioning artifacts and biases introduced by non-neuronal elements or structural features. The sectioning artifact is most pronounced in Vibratome-sectioned tissue and may be described as "chatter." This artifact has the appearance of evenly spaced ridges of tissue that are oriented parallel to the edge of the sectioning blade and are most likely a product of both the vibration and sharpness of the Vibratome blade. The presence of these ridges introduces the possibility of uneven tissue penetration by the antibody, and hence a bias toward a disjunctive distribution of immunoreactive cells. Although it is possible to minimize this effect, it is our experience that it can rarely be eliminated. To insure that this factor did not bias the results of the present study, several controls were performed. First, although all blocks were sectioned in the frontal plane, the orientation of the blade edge and the direction of cutting were varied between cases, as well as between blocks in monkeys in which multiple blocks were prepared. For example, one block might be sectioned with the blade edge oriented parallel to the principal sulcus and with the direction of sectioning along the dorsal/ventral axis, while another might be sectioned with the blade perpendicular to the plane of the sulcus, with the direction of sectioning going from lateral to medial. In addition to these precautions for Vibratome-sectioned material, some blocks were frozen-sectioned. Sections cut on a freezing microtome generally do not contain this artifact and also exhibit enhanced penetration of immunoreagents. Finally, sections from a number of blocks were incubated in the presence of $0.1-0.3 \%$ Triton X-100 to enhance 


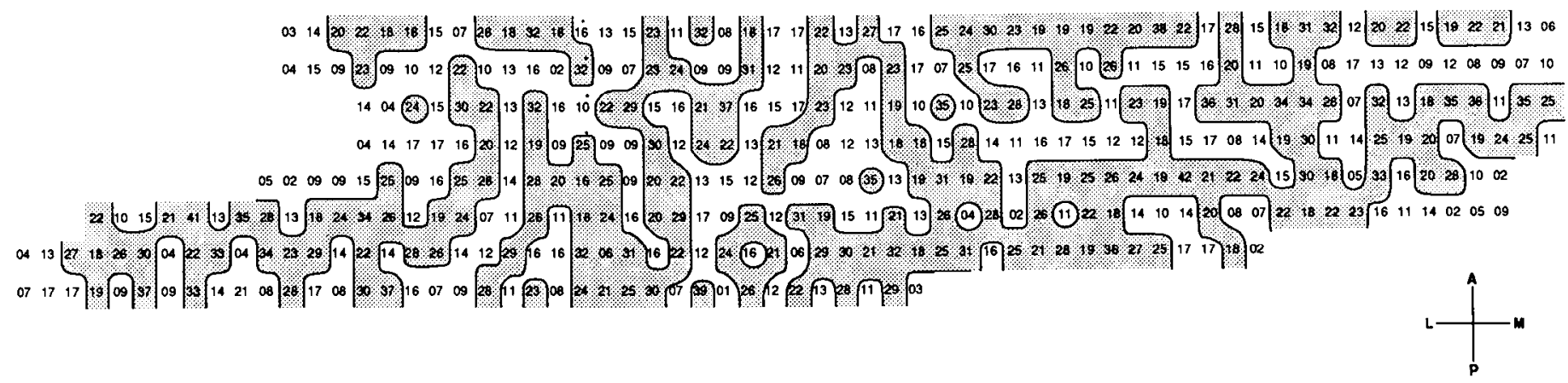

Figure 8. Surface reconstruction of 8 serial sections from the ventral bank of the principal sulcus. Numbers in the reconstruction represent the number of cells with GABA-like immunoreactivity in each $50 \mu \mathrm{m}$ bin. The 8 serial $50 \mu \mathrm{m}$ sections are represented as the 8 lines of bins running in the anterior-to-posterior direction. Sections were aligned using shared blood vessels and surface landmarks. A total of $7771 \mathrm{GABA}-\mathrm{immunoreactive}$ neurons are represented, with a mean of $17.99 / \mathrm{bin}$. Shaded areas represent the connecting of adjacent bins (diagonally, horizontally, and vertically) containing more than the mean number of GABA cells. At intersections where 2 diagonally adjacent bins contain more than the mean number of cells and the remaining 2 contain less than the mean number, the decision as to whether to connect the low or high bins was based on which pair was closest in value. The 2 closest in value were always connected. $A$, anterior; $P$, posterior; $M$, medial; $L$, lateral.

reagent penetration. Analysis of sections treated under these various conditions revealed no differences in either the presence or the periodicity of the pattern of cells reported here.

The second potential source of bias is related to the influence that non-neuronal elements or structural features may have on the distribution of immunoreactive cells. For example, at the surface of a block, penetrating blood vessels that run pcrpendicular to the pial surface may artificially create a region of low cell density. This problem is of particular importance for immunocytochemical studies in which the penetration of immunoreagents is limited to a few superficial microns of tissue. Although the use of penetration-enhancing methods helps to minimize this effect, this source of bias cannot be completely eliminated. The influence of this factor on the distribution of GABA-containing cells is illustrated in Figure 5, where we have marked with asterisks those bins in which large, penetrating vessels (extending at least through layers I, II, and superficial III) were found at one of the surfaces of the section. It is obvious that these vessels have a fairly regular spacing, which corresponds to those bins containing the fewest number of GABAlike cells. Interestingly, nearly every bin containing a large, penetrating blood vessel, and thus a low density of cells, is flanked on eithcr side by bins containing more than the mean number of GABA-like cells. Preliminary data from 2 monkeys in which the brain was sectioned tangential to the pial surface indicate that in the most superficial sections, GABA cell density is greatest surrounding large vascular elements. This raises the possibility that the distribution of GABA-like cells may be related to the organization of vascular elements of the cortex, or, alternatively, that factors such as the diffusion of fixative from these elements may alter the sensitivity of immunohistochemical methods for the detection of certain antigens in various regions of the cortex. In spite of the potential influence of this factor, it alone cannot account for the multiple frequency components reported in the present study, since the distribution of large, penetrating vessels appears to be regularly repeating, with a vessel approximately every $500 \mu \mathrm{m}$, while the high- and lowfrequency components of the GABA distribution have cycles of $150-250$ and $1000-1275 \mu \mathrm{m}$, respectively.

\section{Relationship to previous studies}

Although several studies have noted a "patchy" distribution for GAD (a synthetic enzyme for GABA)-immunoreactive puncta in various regions of monkey sensory cortex (Hendrickson et al., 1981; Houser et al., 1983), to our knowledge this is the first report of a periodic tangential organization for GABA- or GADcontaining somata in any area of the cortex of normal animals, suggesting that this element of intrinsic cortical architecture is hard-wired (however, see Hendry and Jones, 1986, for the effects of visual dcprivation). The failure of previous studies to recognize this feature may be due to several factors. First, the irregular distribution of GABA-containing cells is difficult to discern in sections examined qualitatively, which is perhaps due in part to the large number of GABA-containing cells in all areas of the cortex. This high density provides a basal level of GABA cells upon which the more subtle variations in density are superimposed, making visual resolution of these differences difficult. Although there are no comparable quantitative studies of the tangential distribution of GABA-like neurons for other areas of cortex, data from the study of Houser et al. (1983) are relevant. They counted the number of GAD-positive cells present in a single $430-\mu \mathrm{m}$-wide bin for 7 different sections through areas $3 b$ and $1-2$ of the monkey cortex and found large differences in the number of GAD-positive neurons per bin among the 7 sections examined. They attributed these differences to variability in immunostaining. However, as the positions of the bins were not aligned between sections, an alternative explanation suggested by the present findings is that they may have fortuitously sampled regions of low and high GABA cell density in the different, nonadjacent sections.

A second factor influencing the visualization of this pattern is the sensitivity of the method used to examine GABA-containing cells (Kisvarday ct al., 1986). Previous studies of GABAergic cells in monkey cerebral cortex have used either the uptake of ${ }^{3} \mathrm{H}$-GABA or GAD immunocytochemistry. Both methods have limitations that restrict their ability to demonstrate this irregular tangential pattern. Studies using ${ }^{3} \mathrm{H}-\mathrm{GABA}$ (Hendry and Jones, 1981; Kisvarday et al., 1986) have a very limited region of effective uptake, and thus a limited area over which they can be examined for variations in the tangential distribution of the labeled population. Further, irregularities in such a distribution may also be interpreted as artifacts, owing to the lack of uniformity in the region of uptake. Investigations using GAD antisera (Hendrickson et al., 1981; Houser et al., 1983) are limited by the small number of somata that are immunostained with these antisera. Although this number may be en- 
hanced by colchicine pretreatment, interpretation is once again limited by uncertainties regarding the area, diffusion gradient, and uniformity of the effective colchicine treatment. In contrast to the GAD antisera, the GABA antiserum used in the present study provides good staining of GABA somata without colchicine pretreatment, a property that is shared with other antisera directed against GABA (Seguela et al., 1984; Gabbott and Somogyi, 1986). Furthermore, single-labeling studies comparing immunohistochemical staining that use antisera to either GABA or GAD suggest that they recognize the same ncuronal populations (Hendrickson et al., 1985; Nishimura et al., 1985; Schwartz et al., 1985). This observation is further supported by the double-labeling study of Somogyi et al. (1984), which found that all neurons immunoreactive using antisera to GAD could also be immunostained with antisera for GABA.

Finally, the periodic distribution of GABA-containing cells found for the prefrontal cortex of the monkey in the present study may represent a species or areal specialization of this local circuit population. We consider this alternative unlikely, given the many similarities in modular organization in different cortical areas, including area 46. However, should GABA periodicity turn out to be a special feature of the prefrontal cortex, this would be most interesting. Quantitative analyses of GABA, similar to that carried out here, are needed for other areas of cortex to determine if the periodicity of inhibitory neurons is a general feature of all cortical areas.

\section{Low-frequency component of $G A B A$ cell distribution}

The disjunctive tangential distribution of GABA-containing neurons suggests that the principle of modular organization recognized for physiologically defined properties (Bruce and Goldman-Rakic, 1984) and for afferents and efferents of the association cortex (Goldman and Nauta, 1977; Leichnetz, 1980; Goldman-Rakic and Schwartz, 1982; Schwartz and GoldmanRakic, 1984) may also apply to certain of its local circuit neurons. The distribution of GABA-containing cells described by our spectral analysis is characterized by 2 patterns of organization; a high-frequency component, repeating every 150-250 $\mu \mathrm{m}$, superimposed upon a low-frequency component with a period of roughly $1000-1275 \mu \mathrm{m} /$ cycle. The cycle of $1000-1275$ $\mu \mathrm{m}$, which contains both a high-density and a low-density region of cells with GABA-like immunoreactivity, is remarkably similar to the width of a set of cortical efferent or afferent columns in a number of regions (Jones et al., 1975; Goldman and Nauta, 1977; Bugbee and Goldman-Rakic, 1983; Schwartz and Goldman-Kakic, 1984; Caminiti et al., 1985). Surface reconstructions of the various cortical efferent and afferent columns have shown them to form bands aligned in specific orientations within a given cytoarchitectonic field (Jones et al., 1975; GoldmanRakic, 1984; Caminiti et al., 1985). It is tempting to speculate that the low-frequency component of the GABA distribution represents a similar network of bands, which may be related to the extrinsic connections. For example, variability in the GABA cell distribution might be specifically related to the callosal or, on the other hand, to the associational inputs that are disjunctively distributed and interdigitated in the PS (Goldman-Rakic and Schwartz, 1982). This is a particularly intriguing possibility, since layer IV contains the highest density of GABA-containing cells of any layer and is the principal layer of termination of corticocortical (Goldman-Rakic and Schwartz, 1982; Schwartz and Goldman-Rakic, 1984) and thalamocortical (Giguere and
Goldman-Rakic, 1985) fibers in the prefrontal cortex of the monkey.

In addition to the columnar organization of afferent and efferent domains in the monkey prefrontal cortex, recent 2-deoxyglucose studies have revealed columnar regions of high and low metabolic activity in this area during the performance of behavioral tasks (Goldman-Rakic, 1984). The width of these metabolic bands is similar to the half-cycle of the low-frequency component of the GABA-containing cell distribution. Thus, it is conceivable that some of the variation in metabolic activity may reflect the selective density and action of the local circuit neurons in the cortex.

\section{High-frequency component of $G A B A$ cell distribution}

The high-frequency component of the GABA distribution represents a period of $150-250 \mu \mathrm{m}$ for a complete cycle containing a high- and low-density region of GABA-like cells. The prominence of the high-frequency component in our material was to a large extent unexpected, although its dimensions have a precedent in 2 irregularly distributed systems in the visual cortex: orientation columns and cytochrome oxidase patches. The width of regions activated in area 17 by a visual stimulus of a given orientation, as revealed by optical methods for visualizing physiological activity, corresponds closely to the dimensions of lowand high-density regions of GABA-like cells in the present study (Blasdel and Salama, 1986; Grinvald et al., 1986). In addition, considerable physiological evidence indicates that simple "laterally directed" inhibitory interactions over short distances could explain many features of receptive-field structure, including orientation selectivity (Sillito, 1984). It is relevant that Sillito has speculated that a "periodic pattern of inhibitory loci could constitute the predetermined template for all laterally directed inhibitory interactions in the cortex" $(1984$, p. 111). Possibly, the "microcolumnar" organization of GABA-containing cells described here fits the requirements for this lateral inhibition in the cortex, but this remains to be tested. The width of the highfrequency component of GABA cells is also in the range of that seen for cytochrome oxidase patches in the visual cortex, oval patches with dimensions of $150 \times 200 \mu \mathrm{m}$ located in the centers of ocular dominance columns, which recent studies suggest are functionally related to color processing (Horton and Hubel, 1981; Livingstone and Hubel, 1984). Unlike the patches of high cytochrome oxidase activity, the distribution of GABA-like cells revealed in our serial reconstruction is more of a lattice of short bands (Fig. 8). Furthermore, in prefrontal cortex there is neither physiological nor anatomical evidence of a microorganization corresponding to the $125-\mu \mathrm{m}$-wide GABA "columns." However, the present findings may provide a glimpse of a physiological organization yet to be discovered in this region.

\section{References}

Andersen, R. A., C. Asanuma, and W. M. Cowan (1985) Callosal and prefrontal associational projecting cell populations in area $7 \mathrm{a}$ of the macaque monkey: A study using retrogradely transported fluorescent dyes. J. Comp. Neurol. 232: 443-455.

Arikuni, T., M. Sakai, and K. Kubota (1983) Columnar aggregation of prefrontal and anterior cingulate cortical cells projecting to the thalamic mediodorsal nucleus in the monkey. J. Comp. Neurol. 220: 116-125.

Berod, A., B. K. Hartman, and J. F. Pujol (1981) Importance of fixation in immunohistochemistry: Use of formaldehyde solutions at variable $\mathrm{pH}$ for the localization of tyrosine hydroxylase. J. Histochem. Cytochem. 29: 844-850.

Blasdel, G. G., and G. Salama (1986) Voltage sensitive dyes reveal a 
modular organization in monkey striate cortex. Nature 321:579-585.

Bruce, C. J., and P. S. Goldman-Rakic (1984) Columnar organization of callosal connectivity in the macaque frontal eye fields and its relation to elicited eye movements. Soc. Neurosci. Abstr. 10:59.

Bugbee, N. M., and P. S. Goldman-Rakic (1983) Columnar organization of corticocortical projections in squirrel and rhesus monkeys: Similarity in columnar width in species differing in cortical volume. J. Comp. Neurol. 220: 355-364.

Caminiti, R., S. C. Zeger, P. B. Johnson, A. Urbano, and A. P. Georgopoulos (1985) Corticocortical efferent systems in the monkey: A quantitative spatial analysis of the tangential distribution of cells of origin. J. Comp. Neurol. 241: 405-419.

Eccles, J. C. (1984) The cerebral neocortex. A theory of its operation. In Cerebral Cortex, E. G. Jones and A. Peters, eds., pp. 1-36, Plenum, New York.

Emson, P. C., and O. Lindvall (1979) Distribution of putative neurotransmitters in the neocortex. Neuroscience 4: 1-30.

Freund, T. F., K. A. C. Martin, A. D. Smith, and P. Somogyi (1983) Glutamate decarboxylase-immunoreactive terminals of Golgi-impregnated axoaxonic cells and of presumed basket cells in synaptic contact with pyramidal neurons of the cat's visual cortex. J. Comp. Neurol. 221: 263-278.

Gabbott, P. L. A., and P. Somogyi (1986) Quantitative distribution of GABA-immunoreactive neurons in the visual cortex (area 17) of the cat. Exp. Brain Res. 61: 323-331.

Gigucre, M., and P.S. Goldman-Rakic (1985) Disjunctive distribution of mediodorsal thalamic afferents in the prefrontal cortex of rhesus monkeys. Soc. Neurosci. Abstr. 11: 677

Goldman, P. S., and W. J. H. Nauta (1977) Columnar distribution of cortico-cortical fibers in frontal association, limbic and motor cortex of the developing rhesus monkey. Brain Res. 122: 393-414.

Goldman-Rakic, P. S. (1984) Modular organization of prefrontal cortex. Trends Neuroscience 7: 419-424.

Goldman-Rakic, P. S., and M. L. Schwartz (1982) Interdigitation of contralateral and ipsilateral columnar projections to frontal association cortex in primates. Science 216: 755-757.

Grinvald, A., E. Lieke, R. D. Frostig, C. D. Gilbert, and T. N. Wiesel (1986) Functional architecture of cortex revealed by optical imaging of intrinsic signals. Nature 324: 361-364.

Hedreen, J. C., and T. C. T. Yin (1981) Homotopic and heterotopic callosal afferents of caudal inferior parietal lobule in Macaca mulatta. J. Comp. Neurol. 197: 605-621.

Hendrickson, A. E., S. P. Hunt, and J.-Y. Wu (1981) Immunocytochemical localization of glutamic acid decarboxylase in monkey striate cortex. Nature 292: 605-607.

Hendrickson, A. E., M. Ryan, B. Noble, and J.-Y. Wu (1985) Localization of gamma amino butyric acid (GABA)-containing neurons in macaca monkey and human retina. Inv. Ophthalmol. Vis. Sci. (Suppl.) 26: 95 .

Hendry, S. H. C., and E. G. Jones (1981) Sizes and distributions of intrinsic neurons incorporating tritiated GABA in monkey sensorimotor cortex. J. Neurosci. 1: 390-408.

Hendry, S. H. C., and E. G. Jones (1986) Reduction in number of immunostaincd GABAergic neurons in deprived-eye dominance columns of monkey area 17. Nature 320: 750-753.

Horton, J. C., and D. H. Hubel (1981) A regular patchy distribution of cytochrome-oxidase staining in primary visual cortex of the macaque monkey. Nature 292: 762-764.

Houser, C. R., S. H. C. Hendry, E. G. Jones, and J. E. Vaughn (1983) Morphological diversity of immunocytochemically identified GABA neurons in the monkey sensory-motor cortex. J. Neurocytol. 12:617638.

Jones, E. G. (1984) Identification and classification of intrinsic circuit elements in the cortex. In Dynamic Aspects of Neocortical Function, G. M. Edelman, W. E. Gall, and W. M. Cowan, eds., pp. 7-40, Wiley, New York.

Jones, E. G., and S. P. Wise (1977) Size, laminar and columnar dis- tribution of efferent cells in the sensory-motor cortex of primates. J. Comp. Neurol. 175: 113-136.

Jones, E. G., H. Burton, and R. Porter (1975) Commissural and cortico-cortical "columns" in the somatic sensory cortex of primates. Science 190: 572-574.

Jones, E. G., J. D. Coulter, and S. H. C. Hendry (1978) Intracortical connectivity of architectonic fields in the somatic sensory, motor and parietal cortex of monkeys. J. Comp. Neurol. 181: 291-348.

Jones, E. G., J. D. Coulter, and S. P. Wise (1979) Commissural columns in the somatic-motor cortex of monkeys. J. Comp. Neurol. 188 113-136.

Killackey, H. P., H. J. Gould III, C. G. Cusick, T. N. Pons, and J. H. Kaas (1983) The relation of corpus callosum connections to architectonic fields and body surface maps in sensorimotor cortex of new and old world monkeys. J. Comp. Neurol. 219: 384-419.

Kisvarday, Z. F., A. Cowey, A. J. Hodgson, and P. Somogyi (1986) The relationship between GABA immunoreactivity and labelling by local uptake of $\left[{ }^{3} \mathrm{H}\right] \mathrm{GABA}$ in the striate cortex of monkey. Exp. Brain Res. 62: 89-98.

Krnjevic, K. (1974) Chemical nature of synaptic transmission in vertebrates. Physiol. Rev. 54: 418-540.

Leichnetz, G. R. (1980) An intrahemispheric columnar projection between two cortical multisensory convergence areas (inferior parietal lobule and prefrontal cortex): An anterograde study in macaque using HRP gel. Neurosci. Lett. 18: 119-124.

Livingstone, M. S., and D. H. Hubel (1984) Specificity of intrinsic connections in primate primary visual cortex. J. Neurosci. 4: 2830 2835.

Maley, B., and B. W. Newton (1985) Immunohistochemistry of $\gamma$-aminobutyric acid in the cat nucleus tractus solitarius. Brain Res. 330 . 364-368.

Mountcastle, V. B. (1978) An organizing principle for cerebral function: The unit module and the distributed system. In The Mindful Brain. G. M. Edleman and V. B. Mountcastle, eds., pp. 7-50, MIT, Cambridge, MA.

Nishimura, Y., M. L. Schwartz, and P. Rakic (1985) Localization of $\gamma$-aminobutyric acid and glutamic acid decarboxylase in rhesus monkey retina. Brain Res. 359: 351-355.

Peters, A., and T. M. Walsh (1972) A study of the organization of apical dendrites in the somatic sensory cortex of the rat. J. Comp. Neurol. 144: 253-268.

Ribak, C. E. (1978) Aspinous and sparsely spinous stellate neurons in the visual cortex of rats contain glutamic decarboxylase. J. Neurocytol. $7: 461-478$.

Schwartz, M. L., and P. S. Goldman-Rakic (1984) Callosal and intrahemispheric connectivity of the prefrontal association cortex in rhesus monkey: Relation between intraparietal and principal sulcal cortex. J. Comp. Neurol. 226: 403-420.

Schwartz, M. L., D. S. Zheng, and P. S. Goldman-Rakic (1985) Laminar and tangential variation in the morphology and distribution of GABA-containing neurons in thesus monkey prefrontal cortex. Soc. Neurosci. Abstr, 11: 503.

Seguela, P., M. Geffard, R. M. Buijs, and M. Le Moal (1984) Antibodies against $\gamma$-aminobutyric acid: Specificity studies and immunocytochemical results. Proc. Natl. Acad. Sci. USA 81: 3888-3892.

Sillito, A. M. (1984) Functional considerations of the operation of GABAergic inhibitory processes in the visual cortex. In Cerebral Cortex, A. Peters and E. G. Jones, eds., pp. 91-117, Plenum, New York.

Somogyi, P., A. J. Hodgson, A. D. Smith, M. G. Nunzi, A. Gorio, and J.-Y. Wu (1984) Different populations of GABAergic neurons in the visual cortex and hippocampus of cat contain somatostatin- or cholecystokinin-immunoreactive material. J. Neurosci. 4: 2590-2603.

Thrall, T., and L. Engelman (1983) Univariate and bivariate spectral analysis. In BMDP Biomedical Computer Programs, W. J. Dixon, ed., pp. 604-638, U. California Press, Berkeley.

Walker, A. E. (1940) A cytoarchitectural study of the prefrontal area of the macaque monkey. J. Comp. Neurol. 73: 59-86. 